\title{
Nitric oxide levels in exhaled air and inducible nitric oxide synthase immunolocalization in pulmonary sarcoidosis
}

\author{
Y.P. Moodley*, R. Chetty**, U.G. Lalloo*
}

\begin{abstract}
Nitric oxide levels in exhaled air and inducible nitric oxide synthase immunolocalization in pulmonary sarcoidosis. Y.P. Moodley, R. Chetty, U.G. Lalloo. (C)ERS Journals Ltd 1999. ABSTRACT: Cytokines such as tumour necrosis factor- $\alpha$ and interferon gamma are associated with active pulmonary inflammation in sarcoidosis and they upregulate inducible nitric oxide synthase (iNOS). The objectives of this study were to examine iNOS upregulation in sarcoidosis by showing raised exhaled nitric oxide and increased iNOS activity in lung biopsy specimens of these patients utilizing immunohistochemistry.

Exhaled NO was measured by a chemiluminescence analyser in 12 patients with newly diagnosed biopsy-proven sarcoidosis before and after 6 weeks of corticosteroid therapy. Lung biopsy specimens from these patients were subjected to immunohistochemical staining with a specific iNOS antibody.

Exhaled NO was raised in newly diagnosed sarcoidosis (mean \pm SEM): $9.8 \pm 0.4$ versus 4.1 \pm 0.2 parts per billion (ppb) in 21 healthy controls, $p<0.001$; and fell significantly after 6 weeks treatment with corticosteroids to $5.9 \pm 1.4 \mathrm{ppb} ; \mathrm{p}<0.01$. There was no correlation between exhaled NO and other markers of disease activity. Immunohistochemical staining demonstrated iNOS activity in respiratory epithelium and granulomas in patients with sarcoidosis.

Exhaled nitric oxide is raised in patients with active pulmonary sarcoidosis and may be a result of inducible nitric oxide synthase upregulation. The fall in exhaled nitric oxide following corticosteroid therapy may reflect inhibition of inducible nitric oxide synthase in the respiratory epithelium and granulomas.

Eur Respir J 1999; 14: 822-827.
\end{abstract}

\author{
*Respiratory Unit, Depts of Medicine \\ **Pathology, University of Natal, Dur- \\ ban, South Africa.
}

Correspondence: U.G. Lalloo, Respiratory Unit, Dept of Medicine, University of Natal, Private Bag 7, Congella 4013, Durban, South Africa, Fax: 312604565

Keywords: Exhaled nitric oxide inducible nitric oxide synthase nitric oxide sarcoidosis

Received: July 71998

Accepted after revision May 201999

Y.P. Moodley is in receipt of a South African Pulmonology Society and Astra pharmaceutical training fellowship. This project was supported by a research grant from the South African Medical Research Council.
Nitric oxide synthase (NOS), the enzyme responsible for the generation of nitric oxide (NO), exists in three major isoforms. Neuronal NOS (nNOS) and endothelial NOS (eNOS) are expressed constitutively and are responsible for NO production in the basal physiological state. The regulation of nNOS and eNOS is beginning to be understood, and thus far shear stress, hypoxia and interferon gamma (IFN- $\gamma$ ) have been implicated in their upregulation. Their upregulation is not responsible, however, for the major increases in NO production seen in inflammatory states. Inducible NOS (iNOS) is regulated by cytokines and is responsible for increasing NO concentrations during the inflammatory response. The formation of NO from the oxidation of $\mathrm{L}$-arginine to citrulline occurs in various cells in the respiratory tract such as respiratory epithelium, macrophages, neutrophils, vascular endothelium, smooth muscle cells, mast cells and platelets $[1,2]$. NO has several functions in the airways, including vascular tone regulation, anti- and pro-inflammatory effects, neurotransmission and tumour cell lysis $[1,2]$.

Studies have shown that NO measured at the mouth is derived mostly from the lower respiratory tract and can be measured in exhaled air of humans (exhaled NO) [3-5]. Recently, a great deal of interest has been shown in exhaled NO measurements as a noninvasive surrogate marker of airways inflammation, particularly in asthma [6-10].
In untreated asthmatics, the raised levels of exhaled NO reflected the upregulation of iNOS by the cytokine milieu in the airways as borne out by immunohistochemical and in vitro studies [6-10]. NO produced in cystic fibrosis as a result of airways inflammation may be degraded or absorbed by secretions, thus resulting in low to normal levels measured in exhaled air [11]. Data on subjects with progressive systemic sclerosis having interstitial lung disease and pulmonary hypertension have reported lower levels of exhaled NO compared to those subjects without pulmonary hypertension, suggesting that endothelial dysfunction may impair NO production $[12,13]$. These studies clearly illustrate that NO production may reflect airway inflammation but its measurement in exhaled air is complicated by the underlying pathogenesis of different disease states. It is possible that iNOS activity may differ within such disease states and may be subject to the complications of severity and extent of the disease.

Sarcoidosis is an idiopathic multi-system, granulomatous disorder that predominantly affects the lung, causing endobronchial, peribronchial and parenchymal inflammation. Tumour necrosis factor- $\alpha$ (TNF- $\alpha$ ) and IFN- $\gamma$, cytokines that upregulate iNOS, are raised in active pulmonary sarcoidosis implying that exhaled NO levels should be raised [14-17]. The aim of this study was to measure exhaled NO in patients with newly diagnosed biopsyproven pulmonary sarcoidosis, and to correlate these 
levels with other markers of disease activity such as forced expiratory volume in one second (FEV1), serum angiotensin converting enzyme (SACE) and T-lymphocyte CD4:CD8 ratios in the bronchoalveolar lavage (BAL) fluid. The effect of corticosteroid therapy on exhaled NO after 6 weeks of oral prednisone therapy was investigated, and areas of possible iNOS upregulation identified by immunohistochemical staining of tissue samples obtained from these patients.

\section{Methods}

\section{Subjects}

Study protocols were approved by the university ethics committee and all patients gave informed consent. All patients had biopsy-proven sarcoidosis involving the lung. The characteristics of the study subjects are presented in table 1 .

Twelve patients (6 males) comprised the cohort with newly diagnosed sarcoidosis (10 African and two Asian Indians). Their mean \pm SEM age was $41 \pm 2.1$ yrs. All had constitutional symptoms of fever and fatigue and cough and eight patients presented with dyspnoea. All chest radiographs were abnormal (two Stage I Stilzbach, nine Stage II, one Stage III) and all had high resolution computerized tomography of the chest confirming typical sarcoid interstitial lung disease. Seven patients had associated eye (uveitis) and skin involvement, two patients had skin involvement only and two patients had significant peripheral lymphadenopathy in addition to the lung involvement. SACE levels were raised in 11 subjects to a mean of $76 \pm 26 \mathrm{IU} \cdot \mathrm{mL}^{-1}\left(\right.$ normal $\left.\leq 52 \mathrm{IU} \cdot \mathrm{mL}^{-1}\right)$. Mean FEV1 values were $79.7 \pm 14.5 \%$ of predicted. No patients received glucocorticoids or other immunosuppresive therapy prior to entry into the study.

Healthy controls comprised 21 nonatopic, nonsmoking subjects aged $42 \pm 3.8$ yrs, 10 males with no history of lung disease. None had respiratory tract illnesses in the 6 weeks preceding the study. Poorly controlled steroid naive asthmatics ( $\mathrm{n}=17$; eight males) were used as positive controls in this study.

Table 1. - Characteristics of patients with newly diagnosed active pulmonary sarcoidosis

\begin{tabular}{lccrccc}
\hline $\begin{array}{l}\text { Age } \\
\text { yrs }\end{array}$ & Sex & $\begin{array}{c}\text { FEV1 } \\
\% \text { pred }\end{array}$ & $\begin{array}{c}\text { Baseline } \\
\text { NO } \\
\text { ppb }\end{array}$ & $\begin{array}{c}\text { SACE }^{\#} \\
\text { IU·mL }\end{array}$ & $\begin{array}{c}\text { CD4:CD8 } \\
\text { in BAL } \\
\text { fluid }\end{array}$ & $\begin{array}{c}\text { PA } \\
\text { chest* } \\
\text { film stage }\end{array}$ \\
\hline 41 & F & 78 & 8.1 & 89 & $6: 1$ & II \\
57 & M & 65 & 9.2 & 68 & $5: 1$ & II \\
34 & M & 89 & 8.4 & 69 & $3: 1$ & II \\
54 & F & 97 & 4.0 & 52 & $8: 1$ & II \\
42 & M & 54 & 9.5 & 55 & $4: 1$ & II \\
37 & F & 57 & 10.1 & 150 & $3: 5: 1$ & II \\
28 & M & 87 & 5.6 & 74 & $5: 1$ & III \\
41 & F & 89 & 15.6 & 83 & $5: 1$ & II \\
39 & F & 87 & 18.9 & 68 & $2: 5: 1$ & I \\
37 & F & 71 & 9.8 & 54 & $4: 1$ & II \\
32 & M & 90 & 7.8 & 69 & $5: 1$ & I \\
47 & M & 92 & 9.4 & 74 & $4: 1$ & II \\
\hline
\end{tabular}

M: male; F: female; FEV1: forced expiratory volume in one second; NO: nitric oxide; SACE: serum angiotensin converting enzyme; BAL: bronchoalveolar lavage; PA: postero-anterior view (of the chest radiograph). *: Stilzbach stage of sarcoidosis; ${ }^{\#}$ : normal level $\leq 52 \mathrm{IU} \cdot \mathrm{mL}^{-1}$.

\section{Exhaled nitric oxide measurements}

A Logan R2000 nitric oxide analyser (Logan Research, Rochester, UK) was used. This is a chemiluminescence analyser which is designed for on-line recording of 15,000 parts per billion (ppb) exhaled NO at a resolution of $0.3 \mathrm{ppb}$. It has a response time of $<0.55 \mathrm{~s}$ with high reproducibility. The analyser has a feedback control unit which maintains pressure at $3 \pm 0.4 \mathrm{mmHg}$ and flow at $\sim 1$ $\mathrm{L} \cdot \mathrm{min}^{-1}$ (obstructed) and $5 \mathrm{~L} \cdot \mathrm{min}^{-1}$ (unobstructed)

These parameters do not effect the NO recording. The pressure changes in the mouthpiece and reaction chamber vary insignificantly and causes insignificant changes in NO readings $(<0.1 \mathrm{ppb})$. The sampling rate of the analyser was kept constant at $250 \mathrm{~mL} \cdot \mathrm{min}^{-1}$ for all measurements. The analyser also measures $\mathrm{CO}_{2}$ (response time $200 \mathrm{~ms}$ at a resolution of $1 \% \mathrm{CO}_{2}$ ).

Measurements were made with subjects sitting at least 5 min before testing. The manoeuvre was started with exhalation to residual volume (RV) followed by rapid inhalation to total lung capacity (TLC). Thereafter, the patient exhaled slowly and steadily from TLC over a 30 -s period with an exhalation flow rate of $1 \mathrm{~L} \cdot \mathrm{min}^{-1}$ into a wide-bote Teflon tube connected to the side arm sampler of the analyser. All subjects were allowed several dummy practice manoeuvres. Nose clips were used in the Asian Indian patients and because of recognized technical difficulties (i.e., the nose clips did not provide an adequate seal to prevent nasal contamination of exhaled NO), the African patients were instructed to close their nostrils with their fingers in order to ensure an adequate seal. This precaution was taken, although the contribution from the nasal passages to exhaled NO was not significant and arises mainly from the paranasal sinuses [18].

NO measurements were taken at maximum levels, mean levels and at the level when the $\mathrm{CO}_{2}$ plateau was reached. The latter level represented the alveolar sample of NO as verified by studies in this area and is the value reported in the present study. Corrections for ambient NO levels were made with each measurement.

\section{Transbronchial biopsy and bronchoalveolar lavage}

An Olympus bronchoscope (F1T20D; Olympus, Tokyo, Japan) was inserted and wedged in the right middle lobe with adequate local anaesthesia and sedation, and a segment lavaged using three $60-\mathrm{mL}$ aliquots of saline. The BAL samples were subjected to flow cytometry to establish lymphocyte CD4:CD8 counts. A 1.8-mm size cup biopsy forceps were used to obtain transbronchial specimens. This tissue was transported in formalin and fixed in paraffin wax for histopathology.

\section{Immunohistochemical staining for inducible nitric oxide syndrome}

Lung tissue obtained from patients with sarcoidosis, pulmonary tuberculosis and control subjects were used to identify areas of iNOS activity.

In the group of sarcoidosis patients, transbronchial $(\mathrm{n}=$ $10)$ and open lung biopsies $(n=2)$ were obtained and all specimens underwent immunohistochemical staining. 
Transbronchial biopsy specimens were also obtained from patients with active pulmonary tuberculosis $(n=10)$ confirmed by the presence of acid fast bacilli and caseating granulomas and were used to identify areas of iNOS activity.

The control group was comprised of 10 post mortem lung samples obtained from subjects involved in trauma not involving the lung. Care was taken to exclude any underlying disease state before samples were taken. All the above tissue samples were fixed in paraffin wax before staining for iNOS was performed.

The process of antigen retrieval involved removing the fixative paraffin wax from tissue samples thus exposing antigen sites to immunohistochemical staining with a particular antibody, in this case a specific iNOS polyclonal antibody [19].

Lung biopsies were picked up on adhesive coated slides and incubated at $60^{\circ} \mathrm{C}$ for $10 \mathrm{~min}$. Sections were then passed through two changes of xylene for $5 \mathrm{~min}$ each. This was followed by a passage through two changes of $100 \%$ alcohol for $3 \mathrm{~min}$ and two changes of $95 \%$ alcohol for 3 min each and finally dried for $5 \mathrm{~min}$.

Five slides at a time were placed in a thermoresistant plastic coplin jar $(50 \mathrm{~mL})$ filled with $0.01 \mathrm{M}$ buffered sodium citrate solution $\mathrm{pH}$ 6.0. Slides were processed in an H2500 Microwave processor (Energy Beam Science Inc, Agawam, MA, USA) for $10 \mathrm{~min}$ at $85^{\circ} \mathrm{C}$. Sections were allowed to cool in a coplin jar for $20 \mathrm{~min}$ thereafter. Slides were then washed in two changes of phosphate buffered solution (PBS) pH 7.4 and then allowed to dry. A circle was drawn around tissue sections with a Dako pen (Dako A/S, Copenhagen, Denmark) to form a well for reagents on the slide, in which they can interact with the tissue. Slides were then incubated in $3 \% \mathrm{H}_{2} \mathrm{O}_{2}$ at room temperature for 5 min. Thereafter, slides were immersed in $10 \%$ calf serum albumin to remove background staining by nonspecific proteins. Slides were rinsed in PBS and sections incubated in 40 1:20 $\mu \mathrm{L}$ PBS diluted rabbit primary antibody at room temperature for $10 \mathrm{~min}$. Slides were rinsed in three aliquots of PBS and incubated in $40 \mu \mathrm{L}$ of biotinylated link antibody (Dako LSAB 2 kit, peroxidase KO675; Dako A/ $\mathrm{S})$ at $37^{\circ} \mathrm{C}$ for $10 \mathrm{~min}$. Thereafter, slides were rinsed in two changes of PBS and incubated in $40 \mu \mathrm{L}$ of peroxidaselabelled streptavidin (Dako LSAB 2 kit, peroxidase KO675; Dako A/S) at room temperature for $10 \mathrm{~min}$. Slides were then rinsed twice in PBS and $40 \mu \mathrm{L}$ of a substratechromogen (Dako liquid 3,3-diaminobenzidine (DAB) substrate-chromogen kit K3466; Dako A/S) was added to the slides and incubated for $10 \mathrm{~min}$. Distilled water was then used to rinse the slides and Mayers haematoxylin used to counterstain the slides for $3 \mathrm{~min}$. Slides were then rinsed in $1 \%$ ammonia urate to develop counterstain. After rinsing the slides again, sections were dehydrated through ascending grades of alcohol and cleared by rinses with xylene. Sections were then mounted onto coverslips with DPX mountant (BDH Laboratory Supplies, Poole, UK).

A specific affinity-purified rabbit polyclonal antibody was used to stain for iNOS activity (iNOS 2 (C-19): S1649; Santa Cruz Biotech Inc., Santa Cruz, CA, USA). The procedure was performed according to the protocol set out by the manufacturer. A dilution of 1:20 antibody to PBS was used. Furthermore, negative controls involved immunoabsorption of iNOS antisera with respective antigens before incubation with tissue sections, or incubating tissue sections with normal serum instead of the first layer antiserum.

\section{Statistical analysis}

The data are expressed as mean \pm SEM. Statistical analyses of comparisons were performed using Student's t-test, and for parametric data and analysis of correlation Spearman's rank correlation was used. A p-value $<0.05$ was regarded as significant.

\section{Results}

\section{Exhaled nitric oxide}

Figure 1 shows the comparative NO values between the different groups. The exhaled NO levels were significantly elevated in patients in newly diagnosed sarcoidosis $(9.8 \pm 0.4 \mathrm{ppb})$ compared to the age matched healthy controls $(4.1 \pm 0.2 \mathrm{ppb} ; \mathrm{p}<0.001)$. There was no racial differences in exhaled NO levels both in patients with sarcoidosis and normal healthy subjects. In sarcoidosis patients, levels were 9.2 and $9.5 \mathrm{ppb}$ in the two Asian Indian patients and $9.4 \pm 0.7 \mathrm{ppb}$ in African patients.

Control subjects comprised eight Asian Indian and 13 African subjects. There was no significant difference in exhaled NO levels between Asian Indians (4.5 $\pm 0.4 \mathrm{ppb})$ and Africans $(4.4 \pm 0.6 \mathrm{ppb} ; \mathrm{p}=0.61)$. The four patients not treated with corticosteroids had persistently elevated exhaled NO levels. There was no significant variability in the measurement of exhaled NO levels in normal subjects: $4.1 \pm 0.2 \mathrm{ppb}$ measured on day 1 and $4.5 \pm 0.4 \mathrm{ppb}$ measured on day $3 ; p=0.47$. There was also no significant variability in the measurement of exhaled NO in patients with sarcoidosis: $9.8 \pm 0.4 \mathrm{ppb}$ measured on day 1 and $9.6 \pm 0.7 \mathrm{ppb}$ measured on day $3 ; \mathrm{p}=0.52$.

There was no significant correlation between exhaled $\mathrm{NO}$ and FEV1 $(\mathrm{r}=-0.06)$, SACE $(\mathrm{r}=0.13)$ nor CD4:CD8 ratio on BAL $(\mathrm{r}=0.07)$.

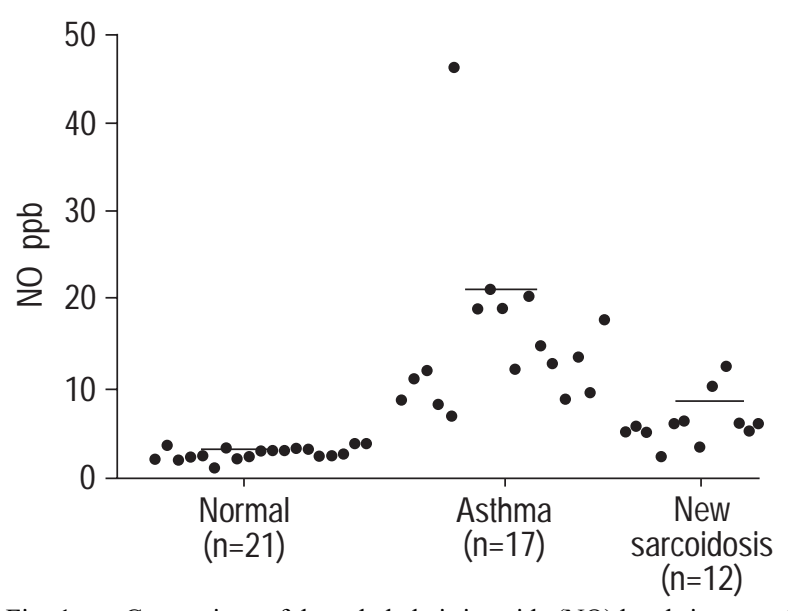

Fig. 1. - Comparison of the exhaled nitric oxide (NO) levels in normal subjects, asthmatics (positive controls) and newly diagnosed sarcoidosis patients; ppb: parts per billion. Horizontal bars represent the mean values; Pre-RX: pretreatment; Post-RX: posttreatment. 


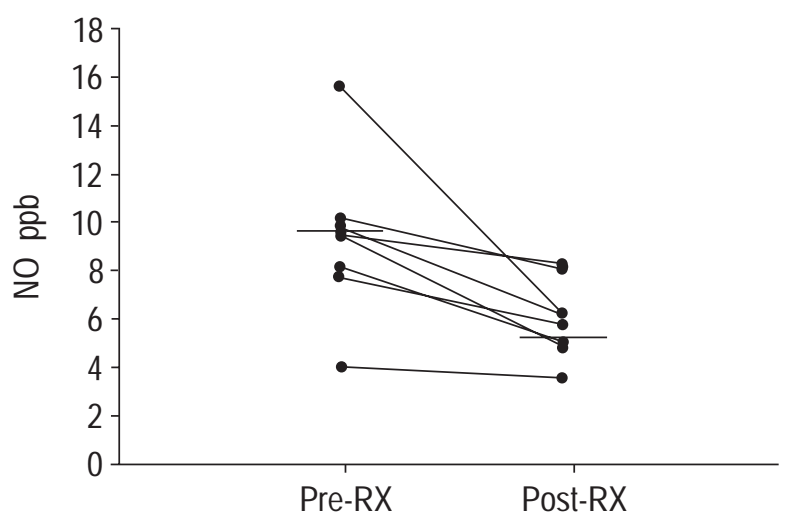

Fig. 2. - Significant fall in exhaled nitric oxide (NO) levels after 6 weeks of corticosteroid therapy in patients with newly diagnosed sarcoidosis $(\mathrm{p}=0.01)$; ppb: parts per billion; Pre-RX: pretreatment; Post$\mathrm{RX}$ : posttreatment. Horizontal lines represent the mean.

Eight patients with newly diagnosed sarcoidosis were treated with corticosteroids. Initial levels of exhaled NO and at 6 weeks after treatment with prednisone $40 \mathrm{mg}$ daily were measured. Figure 2 shows a significant fall in exhaled NO after the treatment period $(9.7 \pm 0.5$ to $5.9 \pm 0.7$ $\mathrm{ppb} ; \mathrm{p}=0.01$ ).

\section{Immunohistochemical staining for inducible nitric oxide synthase}

Figure $3 \mathrm{a}$ is a photomicrograph of normal lung tissue showing scattered staining in the epithelial lining of the respiratory tract and macrophages. In sarcoidosis, the staining was predominantly in the respiratory epithelium and granulomas (fig. 3b). The stain was taken up by macrophages and lymphocytes, as well as fibroblasts. Lymphocytes not involved in granuloma formation did not stain for iNOS. The intracellular staining patterns showed uptake both in the nucleus and cytoplasm of cells, especially macrophages and T-lymphocytes involved in granuloma formation. The intensity of staining for iNOS did not correlate with exhaled NO levels. The transbronchial biopsy specimens were small and may not reflect the extent of disease or location of granulomas, factors that may influence exhaled NO levels.

The transbronchial biopsy specimens from patients with active pulmonary tuberculosis also stained for iNOS activity (fig. 3c). The findings were remarkably identical to that obtained for sarcoidosis. There was increased uptake in the respiratory epithelium and granulomas. Increased staining is demonstrated in the macrophages, T-lymphocytes and fibroblasts, predominantly in the granulomas. The intracellular staining patterns show both a nuclear as well as cytoplasmic uptake.

\section{Discussion}

The present study has shown that exhaled NO is elevated in patients with newly diagnosed active pulmonary sarcoidosis and decreases significantly following 6 weeks of corticosteroid treatment. Immunohistochemical staining demonstrated iNOS in the pulmonary sarcoid granulomas of these patients. Similar staining for iNOS
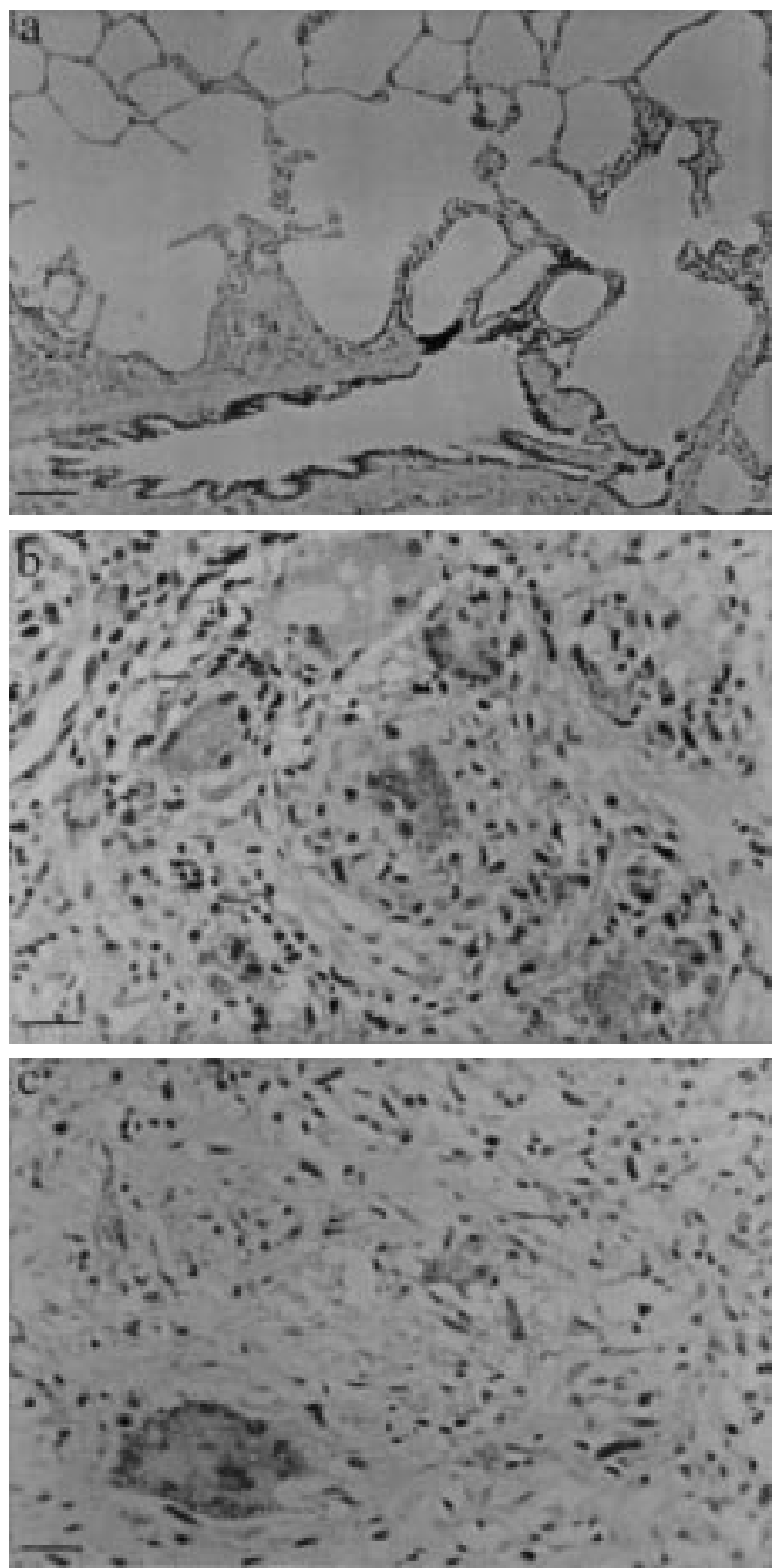

Fig. 3. - a) Immunohistochemical staining for inducible nitric oxide synthase (iNOS) in lung tissue from normal subjects demonstrating uptake in respiratory epithelium and macrophages (brown staining). b) Immunohistochemical staining for iNOS in lung tissue from patients with sarcoidosis demonstrating uptake in respiratory epithelium, macrophages and lymphocytes involved in granuloma formation (brown staining). c) Immunohistochemical staining for iNOS in lung tissue from patients with pulmonary tuberculosis demonstrating uptake in respiratory epithelium, macrophages and lymphocytes involved in granuloma formation (brown staining). Internal scale bars= a) $268 \mu \mathrm{m}$; b) and c) $67 \mu \mathrm{m}$.

was found in the caseating granulomas of lung biopsies of patients with active pulmonary tuberculosis. This suggests upregulation of iNOS in pulmonary granulomatous inflammation and explains elevated exhaled NO observed in active sarcoidosis.

NO is produced endogenously in the human airways (respiratory epithelium, endothelium and macrophages) [1, 2]. It has a variety of physiological functions such as 
vascular smooth muscle relaxation, bacteriostasis and neurotransmission $[1,2,20,21]$. Studies have shown that NO can be measured in exhaled air in physiologically normal as well as pathological states. There are variations in the method of NO measurement and this prompted a standardized technique to be proposed by a European Respiratory Society task force on the measurement of exhaled and nasal nitric oxide measurements [22-25]. The rapid on-line measurement of NO from air continuously exhaled into the chemiluminescence analyser minimizes the loss of NO from the exhaled air. The specifications of this analyser met the proposals laid down by the task group, i.e. response time (lag time $<2 \mathrm{~s}$, rise time $<2 \mathrm{~s}$, total response time $<7 \mathrm{~s})$, resolution $( \pm 1 \%$ full range), measurement range $(0.1-10,000 \mathrm{ppb})$ sample gas flow range $\left(250-706 \mathrm{~mL} \cdot \mathrm{min}^{-1}\right)$. This study also complied with the recommended exhalation pressures (not higher than $20 \mathrm{cmH}_{2} \mathrm{O}$ ) and flow $\left(5-15 \mathrm{~L} \cdot \mathrm{min}^{-1}\right.$ ) during NO measurements as proposed by the task force. The NO measurement taken in this study was the endtidal values corresponding to the plateau of $\mathrm{CO}_{2}$ measured in the exhaled air since this are shown from previous studies to best represent NO produced in lower airways [5].

Exhaled NO in age-matched healthy controls in the present study were similar to reported normal values: $4.1 \pm 0.2 \mathrm{ppb}$ [14]. The immunohistochemical and in vitro studies demonstrating the upregulation of iNOS by the cytokine milieu present in the airways in asthma is reflected by the elevated exhaled NO levels found in these patients [9]. Steroid-naive asthmatics therefore formed positive control subjects in the present study and the elevated exhaled NO $(21.7 \pm 3.6 \mathrm{ppb})$ was similar to that reported in the studies investigating this cohort of patients [7-10].

Exhaled NO was significantly elevated in newly diagnosed sarcoidosis compared to healthy control subjects. Although constitutive isoforms of NOS generate NO and may contribute to baseline levels seen in control subjects, it is less likely that they are responsible for the significant increase in exhaled NO seen in pathological conditions. More studies are needed to verify the role of constitutive NOS in this area. The elevated NO seen in the present study is most likely due to iNOS induction by the cytokines TNF- $\alpha$ and IFN- $\gamma$ which are elevated in active pulmonary sarcoidosis [14-17]. This hypothesis is supported by the positive specific iNOS immunohistochemical staining demonstrated in tissue samples from patients with newly diagnosed sarcoidosis. This is in contrast to tissue from control subjects, which showed slight iNOS activity in respiratory epithelium and occasional macrophages. Lung biopsy tissue from patients with sarcoidosis and active pulmonary tuberculosis stained strongly for NOS in the respiratory epithelium, macrophages and lymphocytes within the granulomas in the present study. Similar iNOS staining was noted in active pulmonary tuberculosis tissue in the study by HwANG et al. [26]. The inflammatory cytokine milieu found in the airway of active pulmonary sarcoidosis raised levels of TNF- $\alpha$ and IFN- $\gamma$, most likely upregulates iNOS activity in these granulomatous disorders [14-16].

The lack of correlation between exhaled NO levels and conventional markers of disease activity suggests that iNOS induction is a response to the inflammatory pro- cesses of sarcoidosis in the airways and may not reflect the granulomatous burden nor the structural changes taking place in the lung. Interestingly, one study has shown no correlation between TNF- $\alpha$ levels and forced vital capacity or alveolitis [27]. The fall in exhaled NO in patients with sarcoidosis treated for 6 weeks with corticosteroids most likely represents downregulation of iNOS and is probably similar to that reported in asthma following oral corticosteroids [28].

A recent study has shown that exhaled NO was not raised in Irish patients with active newly diagnosed pulmonary sarcoidosis [29]. This difference is not explained by the methodology used in measuring NO levels in exhaled air as both methods were similar, but the cohort of patients investigated may well be different. Sarcoidosis usually runs a more indolent and chronic course and tends to present late in the patients in the current clinical setting. Under these circumstances, significant iNOS induction may occur reflecting the elevated NO levels in exhaled air and strongly positive iNOS staining within granulomas.

O'DonNELl et al. [29], in their study, state that they "cannot answer the question whether excess NO generation occurs in Black patients. This disease tends to follow a course with more frequent end-organ damage than that of Caucasian patients". The present study demonstrates increased NO generation in African patients with sarcoidosis.

The NO levels in exhaled air of patients with sarcoidosis was not invariably raised (three out of 12 patients had normal levels) nor were the levels as high as in asthma. The subepithelial location of granulomas, a potential source of $\mathrm{NO}$, may result in impaired diffusion of NO into the airways in contrast to asthma where the largest source of NO is the respiratory epithelium and may also explain the variable levels in sarcoidosis.

Further verification is required for the strong nuclear staining by macrophages and T-lymphocytes within granulomas since iNOS is a predominantly cytoplasmic enzyme, though nuclear staining does occur. It is possible that iNOS activity is present along the nuclear membrane during upregulation by cytokines in pulmonary tuberculosis and sarcoidosis. It is less likely that the antibody used for iNOS staining cross-reacted with a substance in the nucleus since this is a specific antibody for iNOS and that staining for iNOS in healthy control subjects in the current study has shown staining patterns in keeping with previous immunohistochemical studies of iNOS in the lung [30, 31].

The observation of lymphocytes within granulomas staining for iNOS and those in surrounding tissue not staining is interesting. It is possible that stimuli inducing lymphocytes to participate in granuloma formation may also be the factors upregulating iNOS. An alternative explanation is that these are two independent processes.

This study has shown that the upregulation of inducible nitric oxide synthase in sarcoidosis most probably explains the raised levels of exhaled nitric oxide. The subsequent fall in exhaled nitric oxide after corticosteroid therapy may reflect inducible nitric oxide synthase inhibition by this drug in patients with active sarcoid alveolitis.

Acknowledgements. The authors would like to thank P. Padayachee for assistance with the iNOS staining procedures. 


\section{References}

1. Barnes PJ, Belvisi MG. Nitric oxide and lung disease. Thorax 1993; 48: 1034-1043.

2. Gaston B, Drazen JM, Losealzo J, Stamler JS. The biology of nitrogen oxides in the airways. Am J Respir Crit Care Med 1994; 149: 538-551.

3. Gustafsson LE, Leone AM, Persson G, Wiklund MP, Moncada S. Endogenous nitric oxide is present in the exhaled air of rabbits, guinea pigs and humans. Biochem Biophys Res Commun 1991; 181: 852-857.

4. Borlan C, Cox Y, Higenbottam T. Measurement of exhaled nitric oxide in man. Thorax 1993; 48: 1160-1162.

5. Byrnes CA, Dinarevic C, Bush A, Shineboume EA. Is nitric oxide in exhaled air produced at airway or alveolar level? Eur Respir J 1997; 10: 1021-1025.

6. Kharitonov SA, Fan Chung K, Evans D, O'Connor BJ, Barnes PJ. Increased exhaled nitric oxide in asthma is mainly derived from the lower respiratory tract. $\mathrm{Am} \mathrm{J}$ Respir Crit Care Med 1996; 153: 1773-1780.

7. Alving K, Weitzberg E, Lundberg J. Increased amount of nitric oxide in exhaled air of asthmatics. Eur Respir $J$ 1993; 6: 1268-1270.

8. Kharitonov SA, Yates D, Robbins A, Logan Sinclair R, Shinebourne E, Barnes PJ. Increased nitric oxide in exhaled air of asthmatic patients. Lancet 1994; 343: 146147.

9. Hamid Q, Springall DR. Riveros-Moreno V. Induction of nitric oxide synthase in asthma. Lancet 1993; 342: 15101513.

10. Kharitonov SA, Yates DH, Barnes PJ. Inhaled glucocorticosteroids decrease nitric oxide in exhaled air of asthmatic patients. Am J Respir Crit Care Med 1996; 153: 454-457.

11. Balfour-Lynnx IM, Laverty A, Dinwiddie R. Reduced upper airway nitric oxide in cystic fibrosis. Arch Dis Child 1996; 75: 319-322.

12. Kharitonov SA, Cailes JB, du Bois RM, Barnes PJ. Decreased Nitric Oxide in the exhaled air of patients with Systemic Sclerosis with pulmonary hypertension. Thorax 1997; 52: 1051-1055.

13. Cailes JB, Kharitonov SA, Yates D, Barnes PJ, Du Bois RM. Decreased endogenous nitric oxide in the exhaled air of systemic sclerosis patients. Thorax 1995; 50: 449-452.

14. Boros DL. Granulomatous inflammation. Prog Allergy 1978; 24: 183-267.

15. Adams DO. The granulomatous inflammatory response. A review. Am J Pathol 1976; 84: 164-191.

16. Ziegenhagen MW, Benner UK, Zissel G, Zabel P, Sihlaak M, Muller-Quernheim J. Sarcoidosis: TNF- $\alpha$ release ftorn Alveolar Macrophages and Serum Level of sIL2R are Prognostic Markers. Am J Respir Crit Care Med 1997; 156: $1586-1592$.
17. Menshall EM, Tsicopoulos A, Yasruel Z, et al. Cytokine mRNA gene expression in active and nonactive pulmonary sarcoidosis. Eur Respir J 1997; 10: 2034-2039.

18. Lundberg JON, Rinder J, Weitzberg E, Lundberg JM, Alving K. Nasally exhaled nitric oxide originates mainly in the paranasal sinuses. Acta Physiol Scand 1994; 152: 431-432.

19. Noton AS. Microwave oven heating for antigen unmasking in routinely processed tissue sections. J Pathology 1993; 71: 79-80.

20. Nathan C, Xie QW. Nitric oxide synthases: roles, tolls and controls. Cell 1994; 78: 915-916.

21. Nathan C, Yie QW. Regulation of biosynthesis of nitric oxide. J Biochem 1994; 269: 13725-13728.

22. Kharitonov S, Alving K, Barnes PJ, ERS Task Report. Exhaled and nasal nitric oxide measurement: recommendation. Eur Respir J 1997; 10: 1683-1693.

23. Barnes PJ, Kharitonov SA. Exhaled nitric oxide, a new lung function tests. Thorax 1996; 51: 233-237.

24. Robbins RA, Floreani AA, Von Essen SG. Measurement of exhaled nitric oxide by three different techniques. Am J Respir Crit Care Med 1996; 1533: 1631-1635.

25. Kharitonov SA, Robbins RA, Yates D, Keatings V, Barnes PJ. Acute and chronic effect of cigarette smoking on exhaled nitric oxide. Am J Respir Crit Care Med 1995; 152: 609-612.

26. Hwang KH, Wang CH, Liu CY, Yu CT, Kuo HP. Upregulation of inducible nitric oxide synthase from alveolar macrophages and increased level of exhaled nitric oxide in patients with active tuberculosis (abstract). Am J Respir Crit Care Med 1997; 155: A335.

27. Peuringer RJ, Shwartz RA, Dayton GS, Gilbert SR, Hunninghake GW. The relationship between alveolar macrophage TNF, IL-1 and PGE $_{2}$ release, alveolitis and disease severity in sarcoidosis. Chest 1993; 103: 832-838.

28. DiRosa M, Radomski M, Carnuccio P, Moncada S. Glucocorticosteroids inhibit the induction of nitric oxide synthase in macrophages. Biochem Biophys Resp Commun 1990; 172: 1246-1252.

29. O'Donnell DM, Moynihan J, Finlay GA, Keatings VM, McLoughlin P, Fitzgerald MX. Exhaled nitric oxide and bronchoalveolar lavage nitrite/nitrate in active pulmonary sarcoidosis. Am J Respir Crit Care Med 1997; 156: 18921896.

30. Kobzik L, Bredt DS, Lowenstein CJ, et al. Nitric oxide synthase in human and rat lung: immunocytochemical and histochemical localisation. Am J Respir Cell Mol Biol 1993; 9: 371-377.

31. Saleh D, Ernest P, Lim S, Barnes PJ, Giaid A. Increased formation of potent oxidant peroxynitrate in the airways of asthmatic patients is associated with induction of nitric oxide synthase: effect of inhaled glucocorticoid. FASEB J 1998; 12: 929-937. 